\title{
021 IMPAIRED DECISION-MAKING AND DIFFUSION ORIENTATIONAL COMPLEXITY IN PEOPLE WITH MULTIPLE SCLEROSIS
}

doi:10.1136/jnnp-2013-306103.21

N Muhlert, V Sethi, M Ron, L Cipolotti, G Parker, HA Haroon, T Yousry, CA Wheeler-Kingshott, DH Miller, DT Chard. NMR Research Unit, Department of Neuroinflammation, UCL Institute of Neurology, London, WC1N 3BG, UK

Objective Difficulties with decision-making have been reported in people with multiple sclerosis (MS). It is however unclear what aspect of decision making is impaired, for example whether they are more impulsive, and how any impairments relate to grey matter pathology. In this study we assess grey matter microstructure using a novel measure of the number of diffusion orientations on diffusion MRI "diffusion orientation complexity (DOC)". We studied DOC in cortical areas known to be associated with decision making and looked at its associations with performance on the Cambridge Gambling Task (CGT).

Method One hundred and five patients with MS (61 RR, 26 SP, 18 PP; mean age: 45.9 years) and 36 healthy controls (mean age: 39.6 years) were studied. Decision making performance was assessed using the CGT. T1-weighted (T1w) scans $(1 \mathrm{x} 1 \mathrm{x} 1 \mathrm{~mm})$ and cardiac-gated diffusion scans $(2 \times 2 \times 2 \mathrm{~mm}, 61$ directions at $\mathrm{b}=1200 \mathrm{~s} / \mathrm{mm} 2,7$ at $\mathrm{b}=0$ ) were acquired on a $3 \mathrm{~T}$ system. T1w images were registered to diffusion scans and segmented to extract GM. GM regions with a priori evidence of an association with decision-making (caudate, hippocampus, middle frontal gyrus, anterior cingulate, medial prefrontal cortex; all bilateral) were masked using the Oxford-Harvard template and mean DOC was measured in these GM regions.

Results On the CGT, patients showed less adjustment of bets to account for the level of risk (t-test, $p<0.01)$ and were significantly slower at making decisions $(p=0.01)$. Patients also showed significantly lower DOC in the caudate $(p><0.01)$, the middle frontal gyrus $(p><0.001)$, the anterior cingulate $(p<0.01)$, and the medial prefrontal cortex $(p=0.001)$, and significantly higher DOC in the hippocampus $(\mathrm{p}><0.05)$, relative to controls. In patients, risk adjustment correlated with hippocampal DOC $(\mathrm{r}=-.23, \mathrm{p}><0.05)$, the length of deliberation correlated with medial prefrontal DOC $(\mathrm{r}=-0.22, \mathrm{p}><0.05)$ and the quality of decision-making correlated with DOC in the anterior cingulate $(\mathrm{r}=0.34, \mathrm{p}=0.001) .>$

Conclusion Decision-making deficits in people with MS relate to difficulties in adjusting to levels of risk rather than increased impulsivity. Changes in decision-making correlate with DOC in grey matter regions associated with those functions, suggesting a relationship with abnormal grey matter microstructure, such as loss of neurites, which is known to occur in MS. 